\title{
Time and Cost Efficiency of Autonomous Vehicles in the Last-Mile Delivery: A UK Case
}

\author{
Yuanyuan Huang ${ }^{1}, \&$ Xuan Feng ${ }^{2}$ \\ 1 School of Architecture and Art, Central South University, China \\ 2 Coventry Business School, Coventry University, UK \\ Correspondence: Xuan Feng, Coventry University, Priory Street, Coventry, CV1 5FB, United Kingdom.
}

Received: February 1, 2021

Accepted: February 21, 2021

Online Published: February 23, 2021

doi:10.5539/ibr.v14n3p26

URL: https://doi.org/10.5539/ibr.v14n3p26

\begin{abstract}
:
In recent years, last-mile delivery has become an increasingly important area in the global supply chain. Practically, there has been an increasing worldwide interest in developing the last-mile delivery robots/vehicles to increase the efficiency of the whole supply chain. Theoretically, several researchers have suggested that using autonomous robots brings more efficiency for delivery. However, almost no current studies consider a specific last-mile delivery activity - transport from supermarkets after loading to pick-up stations before unloading. The goal of this study is to investigate whether and how autonomous vehicles/robots can address the issue of cost and time efficiency. Specifically, this research aims at identifying the time and cost structures of using autonomous vehicles for the delivery along the chosen route - a single way from the Sainsbury's supermarket to the Amazon pick-up station at Coventry. Furthermore, the research aims to find whether using autonomous vehicles is more efficient in time and/or cost than using vans with drivers for this route.
\end{abstract}

Keywords: cost analysis, delivery evaluation, last mile, time efficiency, urban logistics

\section{Introduction}

In recent years, last-mile delivery has become an increasingly important area in the global supply chain. This was made possible by the development of e-commerce. A research conducted by McKinsey \& Company (2019) concluded that the biggest driver of the growth of the last-mile delivery is e-commerce, which has shifted market share from the business-to-business (B2B) to the business-to-customer (B2C) segment (Joerss et al., 2016). Besides e-commerce, Industry 4.0 also plays a vital role in the boom of last-mile delivery. Industry 4.0, a German concept of smart production and logistics, aims at comprising energy and resource efficiency, increased productivity, shorten of innovation, and time-to-market cycles. (Hoffmann \& Prause, 2018). Recent developments in the last-mile delivery industry have heightened the need for higher efficiency. And thanks to the development of Industry 4.0, the low-efficiency problem of the last-mile delivery can be dealt with innovative ideas (Ranieri et al., 2018). Central to the creative solutions for improving both time and cost efficiency is the concept of using autonomous vehicles/robots.

Practically, there has been an increasing worldwide interest in developing the last-mile delivery robots/vehicles to increase the efficiency of the whole supply chain. Estonia plays a leading role in this field with its start-up, Starship Technologies, which focuses on providing a promising solution to solve the last-mile delivery problem (Hoffmann \& Prause, 2018). In September 2016, the start-up announced a strategic partnership with Mercedes-Benz Vans, a German truck producer, to develop the 'Robovans' - a truck-based autonomous-robots model for delivery (Estonian World, 2016). This model, indeed, realizes the 'hub and spoke' concept - a well-known standard model in logistics - and creates a smart solution for bridging longer distances of delivery (Hoffmann \& Prause, 2018). China is also an important player in the area of autonomous delivery robots, as the largest e-commerce platform by revenue, JD.com, conducted its first trial in autonomous driving vehicles for last-mile delivery on June 18, 2017, at Renmin University, Beijing. The vehicle delivered about 10 packages in approximately six hours. JD subsequently deployed approximately 60 autonomous driving vehicles for last-mile delivery at Beijing, Xian, and Hangzhou for pilot AI-based package delivery (Xia \& Yang, 2018).

Theoretically, several researchers have suggested that using autonomous robots brings more efficiency for delivery. However, almost no current studies consider a specific last-mile delivery activity - transport from 
supermarkets after loading to pick-up stations before unloading. The goal of this study is to investigate whether and how autonomous vehicles/robots can address the issue of cost and time efficiency. Specifically, this research aims at identifying the time and cost structures of using autonomous vehicles for the delivery along the chosen route - a single way from the Sainsbury's supermarket to the Amazon pick-up station at Coventry. Furthermore, the research aims to find whether using autonomous vehicles is more efficient in time and/or cost than using vans with drivers for this route.

\section{Literature Review}

The literature review mainly presents current studies focusing on the niche fragment of autonomous/self-driving package delivery vehicles that are used for the last-mile delivery in the context of Industry 4.0 and the E-commerce boom worldwide. Section 2.1 introduces the concept of last-mile delivery. This section also provides the main challenges faced by the last-mile delivery and shows why autonomous delivery vehicles are proposed as a potential solution to these challenges. The following sections 2.2 demonstrates the time-efficiency and cost-saving advantages of introducing autonomous vehicles/robots. In addition to its advantages, using autonomous vehicles/robots has several weaknesses, which are elucidated in section 2.3. Finally, section 2.4 summarizes the literature and introduces the nest-step research in this field.

\subsection{The Last-mile Concept}

The last-mile delivery is defined as 'the final haul of a shipment to its end receiver, be it a shop, a business, a facility or a residence in case of home deliveries' (Dablanc et al., 2013). Specifically, under the rising e-commerce environment, the last-mile delivery can be defined as 'the last stretch of B2C parcel delivery to the final consignee who has to take reception of the goods at home or at a cluster/collection point.' (Gevaers et al., 2009). In a nutshell, the last-mile delivery represents the last part of the supply chain where parcels ordered online are delivered from hubs, such as supermarkets and urban warehouses, to final destinations, i.e., customers' hands or self-pickup stations.

A Stanford research identified that one important feature of the last-mile delivery is that it generally involves three parties - customers, merchants, and delivery providers - each with their own set of expectations and challenges (Lee et al., 2016). The customers have an increasingly complex set of expectations regarding the speed, flexibility, security, and cost of delivery (Lee et al., 2016). As for merchants, consumer preferences have grown increasingly important in the formerly business-oriented parcel-delivery market with the rise of e-commerce. Vendors have identified last-mile services as a key differentiator and are working hard to offer the best customer experience possible, especially by reducing delivery times and operation costs (Joerss et al., 2016). However, many retailers cannot provide same-day delivery or store pickup, which are valued by consumers. For delivery providers, their challenges are how to profitably meet the complex expectations of consumers and merchants (Lee et al., 2016). To meet these expectations, providers need to offer flexible service base on technology innovations, and meanwhile, they need to efficiently manage the delivery costs and peak times (Stevens, 2015). Another challenge for providers is how to deliver poorly packaged goods, which cause problems for providers and negatively affect the merchants because they need to bear the cost of return items.

Therefore, the main development direction of the last mile delivery is for merchants and providers to provide an efficient service for customers, to reduce delivery time and operation cost, and to maximize profit. This vision is also one of the important themes that lead to the new idea of the city, the smart city, where an efficient and sustainable use of resources can be created through the development of new mobility systems and services under the background of Industry 4.0 (Ranieri et al., 2018). However, the current last-mile delivery is usually considered to be the most inefficient due to its specificities such as a spatial distribution of relatively small receiving points, demands for more frequent but smaller shipments, delivery time windows, etc. (Slabinac, 2015).

\subsection{Advantages of Delivery Vehicles/Robots}

The aspiration of developing delivery robots is to achieve fast, cheap, and flexible delivery (Lee et al., 2016). As Ahti Heinla, CEO of Starship Technologies - a leading delivery-robots company created by founding engineers of Skype, said, Starship's vision 'revolves around three zeroes - zero cost, zero waiting time, and zero environmental impact. We want to do to local deliveries what Skype did to telecommunications.' (The New York Times, 2015). Furthermore, companies can release the labour-employer conflict and pursue work-life balance through using autonomous vehicles (Feng, 2020) In this section, the following paragraphs summarize several studies that have been conducted to investigate the advantages of time and the cost efficiency if using autonomous delivery vehicles/robots. 


\subsubsection{Time Efficiency}

Recent studies have shown the time efficiency of using autonomous delivery vehicles/robots according to the delay of the delivery process and the accuracy of delivery windows.

In a research conducted by Boysen et al. (2018), they analysed the delay extent of the delivery process of using autonomous delivery vehicles/robots compared with using delivery trucks with drivers, with the delay extent manifested by the number of late deliveries. The researchers introduced a practical autonomous delivery model the 'robots to depot' model (R2D): the driverless delivery vehicles/robots are carried by a platform - i.e., a truck, to get into the intercity releasing site, then the platform releases them to delivery couriers to customers. After delivering the parcels to customers, the autonomous vehicles/robots will go to specific depots for further collecting. The researchers built mathematic models for the number of late deliveries of using the R2D model and compared the calculation results with those of the status quo. The research finding shows that compared with the traditional way of using drivers and deliverymen, the autonomous delivery model considerably decreases the number of late deliveries, and that to reach the same service level as R2D model, at least 3 more times of deliverymen are required if no autonomous vehicles/robots are deployed (Boysen et al., 2018).

As for the time of delivery windows, a qualitative research conducted by Hoffmann and Prause mentions that autonomous delivery provides a 15-to-20 min delivery window as standard - much more precise than the traditional manned delivery that so far is only able to provide a general date (calendar day) beforehand (Hoffmann \& Prause, 2018).

\subsubsection{Cost Efficiency}

Much of the current literature on the cost efficiency of using autonomous delivery vehicles/robots pay particular attention to the manufacturing cost, labour cost, and operation cost. The philosophy of most existing researches on cost efficiency is interpretivism, which emphasizes qualitative analysis rather than quantitative analysis (Ryan, 2018). Their research strategy is documentary research, which refers to using personal and official documents as the original data. Documents include but are not limited to newspapers, publications, even tapes, and computer files (Kiss, 2019).

One cost efficiency is that the investment and manufacturing cost of autonomous vehicles/robots is less than the labour cost of using drivers and deliverymen, but this advantage only exits in developed countries where labour costs are high enough to make the return on investment significant (Joerss et al., 2016). In the same vein, because of the claims of Starship CEO Ahti Heinla that their robots would cost no more than $1 \$$ for each delivery when deployed (CNBC, 2015), Hoffmann and Prause emphasize the competitive cost advantage of autonomous delivery robots - up to 15 times cheaper than traditional delivery services - used for the last-mile delivery in suburbs and areas where the traffic is relatively low (Hoffmann \& Prause, 2018).

Furthermore, a Stanford University team concludes that the lack of a driver helps to reduce operating costs. For example, autonomous vehicles/robots consume less energy because they can run at a more stable speed than vehicles with drivers (Lee et al., 2016).

\subsection{Criticisms of Delivery Vehicles/Robots}

Although considerable advantages, some scholars have raised criticisms of using autonomous vehicles/robots due to its both time and cost inefficiency. Most of these criticisms are qualitative analysis based on secondary information, and these scholars prevalently use the induction methods, i.e., generalizing conclusions from specific events.

The time inefficiency results when vehicles running at low speed on long routes. Firstly, the speed of autonomous vehicles/robots depends on public sentiment and traffic regulations (Joerss et al., 2016). In concern of technology immaturity, most countries require that autonomous vehicles/robots only run in pedestrian areas (pavements) at low speed, i.e., walking speed, due to their low weight (Hoffmann \& Prause, 2018). In reality, Starship's self-driving vehicles are designed for driving on sidewalks with a speed of maximal $6 \mathrm{~km} / \mathrm{h}$, much slower than human-driving delivery trucks (Prause, 2019). Furthermore, because most current cities do not have specific roads for autonomous vehicles/robots, their delivery routes are usually subject to various limitations, which make the paths more complex and thus increase the delivery time (Koopman \& Wagner, 2017).

As for cost inefficiency, Joerss et al. (2016) argue that using autonomous vehicles/robots is cheaper than human in developed countries, but in developing countries, labour costs are more likely to remain low enough to impede any new technology changes in the next five to ten years (Joerss et al., 2016). Besides the labour cost, James and Katsuaki found that the cost of introducing pioneer technologies is usually underestimated due to uncertainty, instability, and unrecognized problems (James \& Katsuaki, 1984). Therefore, the profitability of using 
autonomous delivery would depend on many factors and would need to be assessed on a case-by-case basis (Lee et al., 2016).

\subsection{Research Gap}

For time efficiency, current studies mainly focus on the less waiting time for customers. However, whether autonomous vehicles/robots can save more time than human-driven vans running the same route from a place of dispatch directly to the pick-up station is overlooked. Besides, few studies consider the waste of time due to low speed and complex routes.

For cost efficiency, most scholars conclude that using autonomous vehicles/robots costs less by only comparing the labour cost and the manufacturing cost of vehicles/robots. But the relative emerging costs of training, operation, and maintenance, as well as the cost caused by uncertain social impacts, are less studied. In addition, due to the various innovative models of introducing autonomous vehicles/robots and the complicacy of the last-mile delivery system per se, there are few studies that build a heuristic and systematic cost analysis structure for the holistic last-mile delivery industry or for any unit process or activity in this industry.

Overall, these studies highlight the need for deeper research in areas such as the impact of different speeds and routes on time efficiency and the implicit costs of introducing pioneer technologies. Moreover, further study on the systemic cost structure in the last-mile delivery industry will contribute to better analysis for the whole industry.

This research is devoted to addressing the research gap of the scant consideration on a specific last-mile delivery activity - transport from supermarkets after loading to pick-up stations before unloading - through focusing on time and cost efficiency of using autonomous vehicles for a chosen delivery route at Coventry, UK.

\section{Methodology}

\subsection{Modelling Assumptions and Parameters}

This research builds mathematic models to separately demonstrate the time and cost for using autonomous vehicles and using manned tracks in the single route from the Sainsbury's supermarket to the Amazon pick-up station (S-A), as shown in Figure 1. This route is chosen because it represents a typical situation of last-mile delivery from a supermarket or warehouse to a pick-up station in downtown.

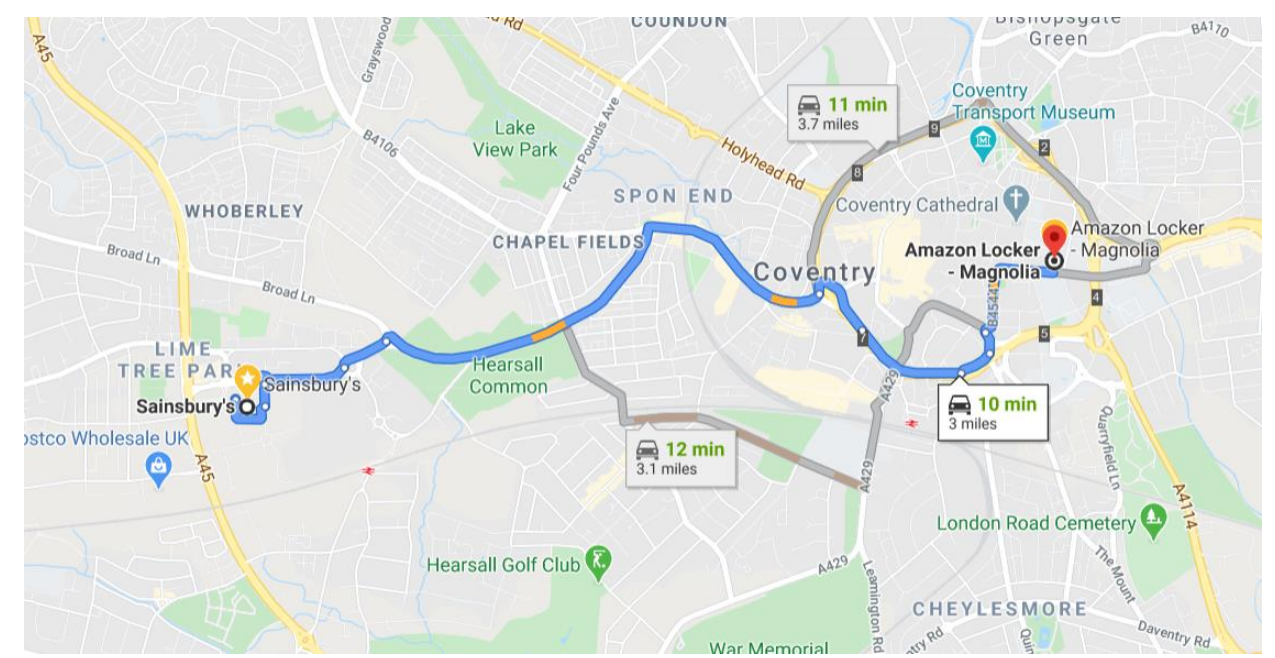

Figure 1. The running route from Sainsbury's to Amazon Locker (Google Map 2020)

\subsubsection{Key Assumptions}

This research establishes the following model assumptions to simplify and idealize the delivery process in the route S-A.

(1) An autonomous vehicle can carry the same weight as a manned truck.

(2) An autonomous vehicle has the same service life as a manned truck.

(3) Each manned truck only needs one driver.

(4) If put into use, one autonomous vehicle can replace one manned truck with one driver. 
(5) Autonomous vehicles run on pedestrian or bike lanes along the motorways at constant low speed. The low speed is required by many governments as delineated in the literature review - section 2.3 (Hoffmann \& Prause, 2018). And because autonomous vehicles are programmed by computers, they can speed up and brake suddenly. They are also easier to run at a constant speed without human behaviours that lead to frequent speed change.

(6) The delivery time is the sum of waiting time at traffic lights and the running time on the road.

(7) The waiting time at traffic lights and the speed of manned trucks are independent variables that are subject to normal distributions.

(8) The waiting time at traffic lights for autonomous vehicles running on pedestrians or bicycle lanes is much shorter than that of manned trucks ( $\mathrm{Li}, 2013)$ and can be idealized to a particle infinitely close to zero in this research.

(9) Student-test (t-test) is appropriately used for verifying the time efficiency of using autonomous vehicles.

(10) For using manned trucks, the delivery cost is mainly determined by the wage of drivers and the energy (gasoline) consumption, and for using manned vehicles, the delivery cost depends mostly on the purchasing cost of unmanned vehicles and the energy (electricity) consumption. While other costs, for example, the maintenance cost, the depreciation cost, the cost of accidents, etc., are the same for both autonomous vehicles and manned trucks, and these costs are low enough to be ignored.

(11) The analysis focus on time and cost efficiency after autonomous vehicles are purchased. Therefore, the purchasing cost of manned trucks is not considered because they are already put into use.

(12) All autonomous vehicles only use electricity, and all manned trucks only use gasoline.

\subsubsection{Delivery Time}

The delivery time (T) of this route is a function of four parameters: the speed of vehicles $(v)$, the distance of the route $(L)$, the number of traffic lights $(n)$, and the waiting time at $i^{\text {th }}$ traffic light $\left(t_{i}\right)$, i.e., $T=f\left(v, L, n, t_{i}\right)$. Thus, the time consumed by manned trucks for the route S-A can be formulated as equation (1), where $\bar{t}$ is the average waiting time at each traffic light, and the subscript $\mathrm{M}$ is for manned trucks.

$$
T_{M}=\sum_{i=1}^{n} t_{M i}+\int_{0}^{L} \frac{d L}{v_{M}} \approx n \times \overline{t_{M}}+\frac{L}{\overline{v_{M}}}
$$

Similarly, the time consumed by autonomous vehicles for route S-A can be formulated as equation (2), where the subscript $\mathrm{A}$ is for autonomous vehicles.

$$
T_{A}=\sum_{i=1}^{n} t_{A i}+\int_{0}^{L} \frac{d L}{v_{A}}=\sum_{i=1}^{n} t_{A i}+\frac{L}{\overline{v_{A}}}
$$

According to assumption (8), the waiting time at each traffic light for autonomous vehicles $\left(t_{A i}\right)$ is much shorter than $\overline{t_{M}}$. Therefore, the accumulated waiting time $\sum_{i=1}^{n} t_{A i}$ can be represented as a positive infinitesimal value $\varepsilon\left(t_{A i}\right)$ that is close to zero, and the equation (2) can be simplified as equation (3)

$$
T_{A}=\frac{L}{v_{A}}+\varepsilon\left(t_{A i}\right)
$$

Where $\varepsilon\left(t_{A i}\right)$ is positive and $\lim _{t_{A i} \rightarrow 0} \varepsilon\left(t_{A i}\right)=0$.

In line with the assumption (7), $t_{M i}$ and $v_{M}$ are independent variables that are subject to normal distributions. The other two parameters $-\mathrm{n}$ and $\mathrm{L}$ - stay the same in equation (1) and (2)/(3) because the distance and the number of traffic lights in the route S-A are constants. This research is devoted to verifying whether $T_{A}$ is significantly less than $T_{M}$. If so, using autonomous vehicles is more time-efficient than using manned trucks. 


\subsubsection{Delivery Cost}

The delivery cost of the route S-A varies from using autonomous vehicles to using manned tracks. For using one autonomous vehicle, the delivery $\operatorname{cost}\left(C_{A}\right)$ mainly comprises the initial cost of purchasing autonomous vehicles $\left(C_{V}\right)$ and the accumulated operating cost of consuming electricity after $m$ deliveries $\left(C_{E}\right)$. Let $C_{E j}$ be the cost of electricity consumption for $j^{\text {th }}$ delivery, and $C_{E j}$ can be expressed as the product of the electricity consumption per distance $(\phi)$, the distance of the route $(L)$, and the electricity price per $\mathrm{kWh}$ for $j^{\text {th }}$ delivery $\left(P_{E j}\right)$, i.e., $C_{E j}=\phi \times L \times P_{E j}$.

By adding $C_{V}$ to $C_{E}$ and simplifying the unit electricity price as a constant value $\overline{P_{E}}$ for each delivery, the research calculates the total delivery cost of each autonomous vehicle through equation (4) and (5).

$$
C_{A}=C_{V}+\sum_{j=1}^{m} C_{E j} \approx C_{V}+\phi \times m \times L \times \overline{P_{E}}
$$

where

$$
C_{E}=\sum_{j=1}^{m} C_{E j}=\phi \times L \times \sum_{j=1}^{m} P_{E j} \approx \phi \times m \times L \times \overline{P_{E}}
$$

While for continue to use manned trucks, the delivery cost $\left(C_{M}\right)$ mainly includes the accumulated wage of drivers $\left(W_{M}\right)$ and the accumulated operating cost of consuming gasoline $\left(C_{G}\right)$. The wage paid a driver for $j^{\text {th }}$ delivery $\left(W_{M j}\right)$ can be gained through multiplying the average wage per hour $\left(\overline{w_{M}}\right)$ by the delivery time for $j^{\text {th }}$ delivery $\left(T_{M j}\right)$, which can be calculated through equation (1). Therefore, the accumulated wage $W_{M}$ is given by equation (6).

$$
W_{M}=\sum_{j=1}^{m} W_{M j}=\sum_{j=1}^{m} \overline{w_{M}} \times T_{M j} \approx m \times \overline{w_{M}} \times \overline{T_{M}}
$$

Where the research uses the average time consumed per delivery $\left(\overline{T_{M}}\right)$ to simplify the calculation.

Similar to $C_{E}, C_{G}$ can be approximately calculated as the product of the gasoline consumption per distance $(\varphi)$, the distance of the route $(L)$, the accumulated number of delivery $(m)$, and the average gasoline price $\left(\overline{P_{G}}\right)$. Therefore, the accumulated cost of gasoline $\left(C_{G}\right)$ is given by equation (7).

$$
C_{G}=\sum_{j=1}^{m} C_{G j}=\varphi \times L \times \sum_{j=1}^{m} P_{G j} \approx \varphi \times m \times L \times \overline{P_{G}}
$$

where $C_{G j}$ and $P_{G j}$ represent the energy cost and the price of gasoline for $j^{\text {th }}$ delivery separately.

Then, $C_{M}$ is the sum of $W_{M}$ and $C_{G}$, as presented in equation (8).

$$
C_{M}=W_{M}+C_{G}=\sum_{j=1}^{m} W_{M j}+\sum_{j=1}^{m} C_{G j} \approx m \times \overline{W_{M}} \times \overline{T_{M}}+\varphi \times m \times L \times \overline{P_{G}}
$$

$C_{A}$ is higher than $C_{M}$ at the beginning of introducing autonomous vehicles because the purchasing price of autonomous vehicles is considerably higher than other operating costs. However, the initial investment on 
autonomous vehicles only happens at the beginning, and $C_{A}$ is assumed to be lower than $C_{M}$ in the long term at the advantage of lower energy consumption and no need to pay for drivers. This research aims to find whether there is a breakpoint $m^{*}$ where $C_{A}$ will be lower than $C_{M}$ after $m^{*}$ deliveries through the single route S-A and to estimate the value of $m^{*}$ if the breakpoint exists.

\subsection{Data Collection}

Data collection and analysis is the last layer and the core of the research onion and can clearly explain the way and purpose of the research (Moser \& Korstjens, 2018). Both primary data and secondary data are used in this research. The primary experiment is appropriate to simulate the waiting time at $i^{\text {th }}$ traffic light $\left(t_{M i}\right)$ and the speed of manned trucks $\left(v_{M}\right)$. Although professional simulation software cannot be used due to COVID19, the two parameters can be represented by random numbers generated by Excel because both the de facto waiting time and the driving speed vary case by case and are, to a large extent, random in reality. Because of these reasons, the values of both the two parameters are gained through separately generating 200 random data subject to normal distributions inside appropriate intervals. Scholars prevalently use the normal distribution to simulate the random data in real life, and 200 data are necessary because big data will create more accurate predictions (Killeen, 2019).

The speed of manned trucks $\left(v_{M}\right)$ has a minimum value of $0 \mathrm{~km} / \mathrm{h}$ at the time of extreme road conditions and a maximum value of $112 \mathrm{~km} / \mathrm{h}$ required by the UK government (GOV.UK, 2020). Based on these constraints, 200 data of $v_{M}$ are generated through Excel, as shown in Figure 2. After using the Minitab software to conduct descriptive statistics analysis for these data, figure 3-4 presents the distribution of manned trucks' average speed - a normal distribution with an average speed of $57.14 \mathrm{~km} / \mathrm{h}\left(\overline{v_{M}}\right)$ and a standard deviation $\left(\sigma_{v_{M}}\right)$ of 25.12, i.e., $v_{M} \sim N\left(57.14,25.12^{2}\right)$.

\begin{tabular}{|c|c|c|c|c|c|c|c|c|c|c|c|}
\hline \multicolumn{10}{|c|}{ Average running speed of manned rucks on route $\mathrm{S}-\mathrm{A}(\mathrm{Km} / \mathrm{h})$} & & \\
\hline 101 & 42 & 40 & 86 & 58 & 29 & 84 & 15 & 76 & 56 & & \\
\hline 20 & 88 & 76 & 57 & 64 & 39 & 53 & 86 & 44 & 32 & & \\
\hline 100 & 53 & 68 & 44 & 7 & 72 & 61 & 78 & 53 & 77 & \multicolumn{2}{|c|}{ Descriptive Statistics } \\
\hline 27 & 28 & 25 & 12 & 51 & 37 & 108 & 63 & 48 & 35 & & \\
\hline 63 & 41 & 83 & 13 & 111 & 80 & 63 & 77 & 58 & 83 & Mean & 57.14 \\
\hline 53 & 99 & 32 & 60 & 27 & 87 & 1 & 52 & 27 & 91 & Standard Error & 1.776168877 \\
\hline 51 & 41 & 49 & 51 & 43 & 58 & 59 & 46 & 29 & 65 & Median & 56 \\
\hline 79 & 56 & 75 & 6 & 67 & 35 & 99 & 51 & 47 & 82 & Mode & 82 \\
\hline 79 & 82 & 39 & 8 & 38 & 72 & 75 & 27 & 85 & 47 & Standard Deviation & 25.1188211 \\
\hline 49 & 76 & 54 & 25 & 23 & 52 & 55 & 39 & 95 & 57 & Sample Variance & 630.9551759 \\
\hline 44 & 96 & 25 & 64 & 36 & 96 & 45 & 3 & 78 & 44 & Kurtosis & -0.57742972 \\
\hline 53 & 37 & 50 & 62 & 70 & 81 & 82 & 111 & 71 & 18 & Skewness & 0.000183263 \\
\hline 74 & 53 & 33 & 61 & 112 & 79 & 24 & 9 & 59 & 55 & Range & 111 \\
\hline 68 & 78 & 81 & 96 & 8 & 57 & 44 & 35 & 69 & 33 & Minimum & 1 \\
\hline 36 & 45 & 51 & 48 & 58 & 75 & 49 & 34 & 59 & 82 & Maximum & 112 \\
\hline 60 & 71 & 59 & 87 & 8 & 93 & 41 & 29 & 82 & 92 & Sum & 11428 \\
\hline 74 & 89 & 67 & 56 & 108 & 36 & 20 & 101 & 62 & 40 & Count & 200 \\
\hline 86 & 55 & 77 & 73 & 82 & 105 & 31 & 70 & 60 & 24 & Confidence Level(95.0\%) & 3.502527841 \\
\hline 75 & 41 & 76 & 65 & 72 & 34 & 30 & 15 & 25 & 55 & & \\
\hline 54 & 56 & 82 & 43 & 102 & 34 & 89 & 35 & 50 & 66 & & \\
\hline
\end{tabular}

Figure 2. Average running speed of manned trucks on route S-A

Similarly, the waiting time at $i^{\text {th }}$ traffic light $\left(t_{M i}\right)$ can also be simulated as a random value from 0 to 2 mins the maximum minutes for waiting (GOV.UK, 2020). The simulation results and the distribution descriptive are presented in Figure 3. The average waiting time $\left(\overline{t_{M}}\right)$ is 1.04 minutes and $t_{M} \sim N\left(1.04,0.52^{2}\right)$. 


\begin{tabular}{|c|c|c|c|c|c|c|c|c|c|c|c|}
\hline \multicolumn{10}{|c|}{ Waiting time at each traffic light for manned trucks (Mins) } & & \\
\hline 0.2 & 0.4 & 0.9 & 0.2 & 0.9 & 1.1 & 1.1 & 1.8 & 1.5 & 1.3 & & \\
\hline 0.4 & 0.3 & 0.6 & 0.2 & 1.7 & 1.9 & 1.9 & 0.9 & 0.2 & 0.6 & & \\
\hline 1.6 & 1.4 & 0.8 & 0.4 & 0.6 & 1.2 & 0.7 & 1.5 & 0 & 1.1 & \multicolumn{2}{|c|}{ Descriptive Statistics } \\
\hline 2.0 & 0.4 & 0.2 & 0.5 & 0.4 & 1.4 & 1.4 & 0.9 & 1.4 & 1.1 & & \\
\hline 1.2 & 1.2 & 0.5 & 1.1 & 0.1 & 1.3 & 1.8 & 1.0 & 2.0 & 0.4 & Mean & 1.041 \\
\hline 1.4 & 1.1 & 0.2 & 0.5 & 1.2 & 1.5 & 0.6 & 1.0 & 0.2 & 1.9 & Standard Error & 0.036965215 \\
\hline 2.0 & 1.0 & 1.0 & 1.4 & 0.7 & 0.6 & 0.3 & 0.8 & 1.3 & 1.1 & Median & 1.1 \\
\hline 0.7 & 1.1 & 1.8 & 1.8 & 1.6 & 0.5 & 1.9 & 1.0 & 0.6 & 1.3 & Mode & 1.1 \\
\hline 1.9 & 0.9 & 1.1 & 0.2 & 1.1 & 1.0 & 0.7 & 0.4 & 0.1 & 1.4 & Standard Deviation & 0.52276709 \\
\hline 1.5 & 0 & 0.6 & 0.7 & 1.3 & 1.3 & 0.4 & 1.1 & 1.6 & 1.2 & Sample Variance & 0.273285427 \\
\hline 0 & 0.3 & 1.1 & 1.8 & 0.8 & 1.5 & 1.0 & 1.9 & 2.0 & 0.7 & |Kurtosis & -0.82284499 \\
\hline 1.6 & 0.5 & 1.7 & 0.7 & 1.7 & 0 & 1.1 & 0.7 & 0.5 & 0.6 & Skewness & -0.10690746 \\
\hline 0.5 & 1.3 & 1.1 & 1.5 & 1.1 & 1.1 & 1.0 & 1.1 & 1.0 & 0.9 & Range & 2 \\
\hline 1.1 & 0.1 & 0.9 & 0.5 & 1.3 & 1.3 & 0.7 & 0.8 & 1.6 & 1.4 & Minimum & 0 \\
\hline 1.1 & 0.6 & 0.5 & 0.4 & 1.0 & 0.9 & 1.6 & 1.6 & 1.3 & 0.8 & Maximum & 2 \\
\hline 1.2 & 1.6 & 1.1 & 1.6 & 1.8 & 2.0 & 0.7 & 1.5 & 0 & 1.0 & Sum & 208.2 \\
\hline 0.1 & 1.5 & 1.3 & 1.0 & 1.1 & 1.1 & 0.4 & 0.8 & 1.8 & 1.4 & Count & 200 \\
\hline 1.1 & 0.4 & 0.5 & 1.0 & 0.7 & 1.4 & 1.0 & 1.3 & 1.3 & 1.2 & Confidence Level(95.0\%) & 0.072893798 \\
\hline 2.0 & 1.2 & 1.7 & 1.9 & 1.1 & 1.6 & 1.6 & 0.8 & 1.4 & 0.2 & & \\
\hline 1.8 & 1.1 & 0.5 & 1.2 & 0.9 & 1.4 & 1.6 & 1.6 & 1.6 & 1.5 & & \\
\hline
\end{tabular}

Figure 3. The waiting time at each traffic lights for manned trucks

The values of other parameters can be obtained through public secondary sources, including websites, journals, Google map, etc. According to Google map, the driving distance of route S-A $(L)$ is 3 miles $(4.8 \mathrm{~km})$, and there are 11 traffic lights located at 11 crossroads.

Due to the law requirements and the technology immaturity, autonomous vehicles are designed to travel on pedestrian and bike lanes at a constant low speed $\left(v_{A}\right)$ of about 4 miles per hour $(6.4 \mathrm{~km} / \mathrm{h})$ (Lee et al., 2016). The average hourly wage of a delivery Driver $\left(\overline{w_{M}}\right)$ in the United Kingdom is $£ 12.78$ (Indeed, 2020). And the estimated price for an autonomous vehicle $\left(C_{V}\right)$ is nearly $£ 24,000$ (Bloomberg, 2020). In the UK, the average electricity price for electro mobiles $\left(\overline{P_{E}}\right)$ is $£ 0.25$ per $\mathrm{kWh}$ (Power Compare, 2020) and the average gasoline price $\left(\overline{P_{G}}\right)$ during the last quarter is $£ 1.23$ per litre, which is calculated based on the UK weekly gasoline price (Global Petrol Prices, 2020), as shown in Figure 4.

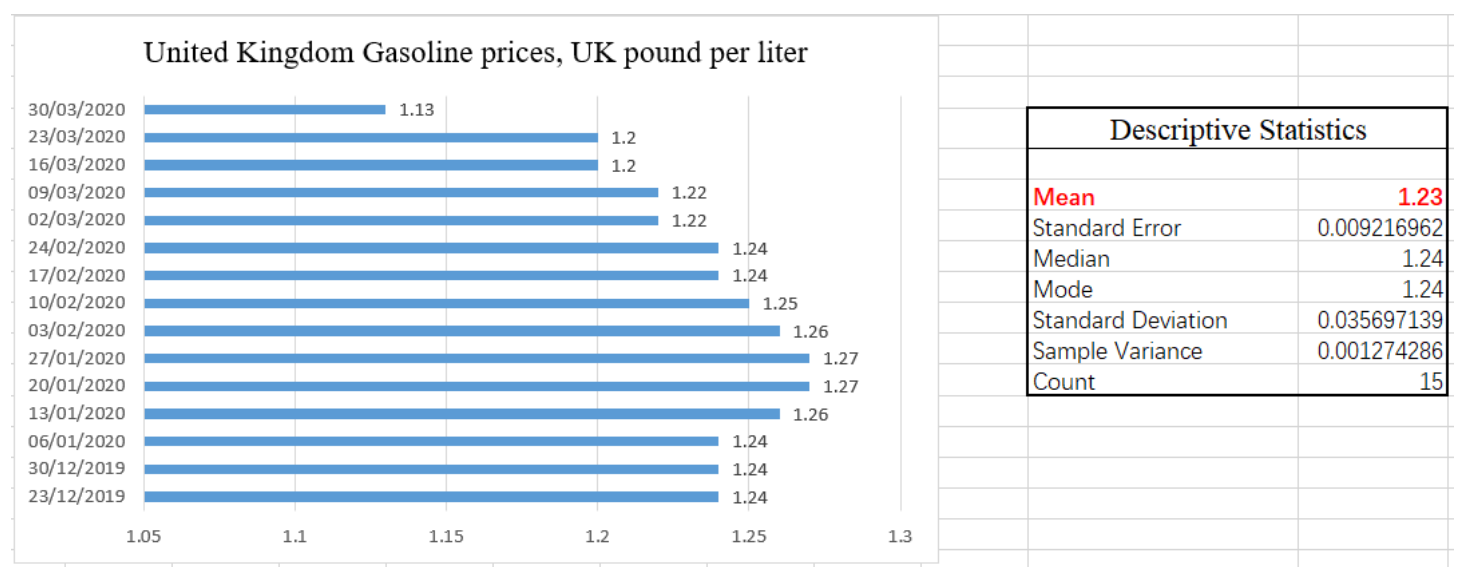

Figure 4. The UK weekly gasoline prices in the first quarter of 2020

Similar to electric cars, electricity consumption $(\phi)$ of autonomous vehicles is $0.19 \mathrm{kWh}$ per kilometre (Aqua-Calc, 2020). While for manned trucks, the gasoline consumption $(\varphi)$ is 0.05 litre per kilometre (Energuide, 2020).

Base on the data collection methods above, Table 1 summarizes the values of parameters used in this research. 
Table 1. Nomenclature of values of parameters

\begin{tabular}{|l|c|l|}
\hline \multicolumn{1}{|c|}{ Parameter } & Symbol & \multicolumn{1}{|c|}{ Value } \\
\hline The distance of route S-A & $L$ & $4.8 \mathrm{~km}$ \\
\hline The number of traffic lights on route S-A & $n$ & 11 \\
\hline The average waiting time at each traffic light for manned trucks & $\overline{t_{M}}$ & $1.04 \mathrm{mins}$ \\
\hline The average speed of manned trucks & $\overline{v_{M}}$ & $57.14 \mathrm{~km} / \mathrm{h}$ \\
\hline The constant speed of autonomous vehicles & $v_{A}$ & $6.4 \mathrm{~km} / \mathrm{h}$ \\
\hline The average hourly wage of a delivery Driver & $\overline{W_{M}}$ & $£ 12.78 \mathrm{per} \mathrm{hour}$ \\
\hline The purchasing price for an autonomous vehicle & $C_{V}$ & $£ 24,000$ \\
\hline The average electricity price for autonomous vehicles & $\overline{P_{E}}$ & $£ 0.25 \mathrm{per} \mathrm{kWh}$ \\
\hline The average gasoline price & $\overline{P_{G}}$ & $£ 1.23 \mathrm{per} \mathrm{litre}$ \\
\hline The electricity consumption of autonomous vehicles & $\phi$ & $0.19 \mathrm{kWh} / \mathrm{km}$ \\
\hline The gasoline consumption of manned trucks & $\varphi$ & $0.05 \mathrm{litre} / \mathrm{km}$ \\
\hline
\end{tabular}

\section{Analysis and Findings}

\subsection{Time Efficiency Analysis}

As mentioned in the methodology chapter, a null hypothesis $\left(H_{0}\right)$ is defined as autonomous vehicles spend less time than manned tracks for the chosen route S-A, i.e., whether the inequation $T_{A}<T_{M}$ holds. To verify $H_{0}$, the hypothesis testing should be used following the decision rule - if the test statistic is greater than the critical value, $H_{0}$ should be refused.

Through putting parameter values in table 1 into equation (3), the time of autonomous vehicles $\left(T_{A}\right)$ can be calculated as equation (9).

$$
T_{A} \approx \frac{L}{v_{A}}+\varepsilon\left(t_{A i}\right)=\frac{4.8 \mathrm{~km}}{6.4 \mathrm{~km} / \mathrm{h}} \times \frac{60 \mathrm{mins}}{\mathrm{h}}+\varepsilon\left(t_{A i}\right)=45 \mathrm{mins}+\varepsilon\left(t_{A i}\right)
$$

Because $\varepsilon\left(t_{A i}\right)$ is positive and $\lim _{t_{A i} \rightarrow 0} \varepsilon\left(t_{A i}\right)=0, T_{A}>45 \mathrm{mins}$.

While for $T_{M}$, because $t_{M i}$ and $v_{M}$ are subject to distributions $\left[v_{M} \sim N\left(57.14,25.12^{2}\right), t_{M i} \sim N\left(1.04,0.52^{2}\right)\right]$, the distribution of $T_{M}$ can be obtained through Minitab software.

For distribution $T_{M} \sim N\left(19.5,6.5^{2}\right)$, the mean value $\left(\overline{T_{M}^{*}}\right)$ is 19.5 , and the standard deviation $\left(\sigma_{T_{M}}\right)$ is 6.5 . These values can help the research to define the test statistic as $=\frac{T_{A}-\overline{T_{M}^{*}}}{\sigma_{T_{M}}}=\frac{45+\varepsilon\left(t_{A i}\right)-19.5}{6.5}>3.92$, which is greater than 3.30 - the critical value to refuse the null hypothesis under the confidence level of $99.9 \%$ and the degree of freedom of 200.

According to the result, the null hypothesis is refused, and the conclusion is that using autonomous vehicles costs more time than using manned trucks under the confidence level of 99.9\%. Therefore, using autonomous vehicles cannot achieve the time efficiency in most cases if the vehicles are limited to run on pedestrian or bike lanes at a low speed of $6 \mathrm{~km} / \mathrm{h}$.

Based on the equation $(2)^{*}-T_{A} \approx \frac{L}{v_{A}}+\varepsilon=\frac{4.8}{v_{A}} \times 60 \frac{\text { mins }}{h}+\varepsilon\left(t_{A i}\right)$, autonomous vehicles should run faster to achieve time efficiency. Using autonomous vehicles can spend the same time as manned trucks when $T_{A} \approx \frac{4.8}{v_{A}}+$ $\varepsilon\left(t_{A i}\right)=\overline{T_{M}^{*}}=19.5$ mins, i.e., $v_{A} \approx 15 \mathrm{~km} / \mathrm{h}$. Using autonomous vehicles is time-efficient only when the test 
statistic $\tau=\frac{T_{A}-\overline{T_{M}^{*}}}{\sigma_{T_{M}}}$ less than $-1.96-$ the critical value to accept the null hypothesis that autonomous vehicles cost less time than manned trucks under the confidence level of $95 \%$. The sufficient speed $v_{A}$ can be calculated through inequation $=\frac{T_{A}-\overline{T_{M}^{*}}}{\sigma_{T_{M}}^{*}}=\frac{\frac{4.8}{v_{A}} \times 60+\varepsilon\left(t_{A i}\right)-19.5}{6.5}<-1.96$, and the result is $v_{A}>42.6 \mathrm{~km} / \mathrm{h}$. This means that if autonomous vehicles want to achieve the significant advantage of time efficiency against manned trucks, the speed of autonomous vehicles should be greater than $42.6 \mathrm{~km} / \mathrm{h}$. And their speed cannot be lower than $15 \mathrm{~km} / \mathrm{h}$, below which the autonomous vehicles will cost more time than manned trucks. While for the speed interval from $15 \mathrm{~km} / \mathrm{h}$ to $42.6 \mathrm{~km} / \mathrm{h}$, autonomous vehicles' time advantage is not very significant.

However, it is not feasible for autonomous vehicles to run on pedestrians or bike lanes at such a high speed over $15 \mathrm{~km} / \mathrm{h}$. If the autonomous vehicles run on motorways, they will also face the time waste due to traffic lights and need to run at speed no less than the average speed of manned trucks $\left(\overline{v_{M}}\right)$, i.e., $57.14 \mathrm{~km} / \mathrm{h}$ according to the result emerged in table 4-1. This is hard to achieve in the short term because autonomous vehicles are subject to a series of limitations and constraints such as technical immaturity and social unacceptance.

The analysis concludes that autonomous vehicles spend more time than manned trucks for the delivery route of S-A. Autonomous vehicles running on pedestrians or bike lanes can never be more time-efficient than manned trucks because the speed of this kind of autonomous vehicles is highly limited in concern of safety. The only method of achieving time efficiency for autonomous vehicles is to improve their running speed to over 57.14 $\mathrm{km} / \mathrm{h}$. The speed is so high that the autonomous vehicles can only run on current motorways mixed with all kinds of manned cars or on special lanes established for autonomous vehicles. However, autonomous vehicles running on current motorways are not feasible in the short term because they are facing huge technical challenges of being smart enough to accomplish all the driving behaviours of a human on roads. In addition to technical constraints, legal limitations and social risks, such as public acceptance, also hinder the running of autonomous vehicles on motorways together with manned cars. Building special lanes for autonomous vehicles cannot achieve in the short term as well because such infrastructure projects always need long-term planning and construction. Therefore, using autonomous vehicles cannot achieve time efficiency in the short term unless all the technical, legal, and social challenges are adequately addressed.

\subsection{Cost Efficiency Analysis}

The cost of using autonomous vehicles $\left(C_{A}\right)$ can be presented as Equation (10) below through putting values of parameters in Table 1 into equations (4) and (5).

$$
C_{A} \approx C_{V}+\phi \times m \times L \times \overline{P_{E}}=24,000+0.228 m
$$

The curve of Equation (10) is shown in Figure 5.

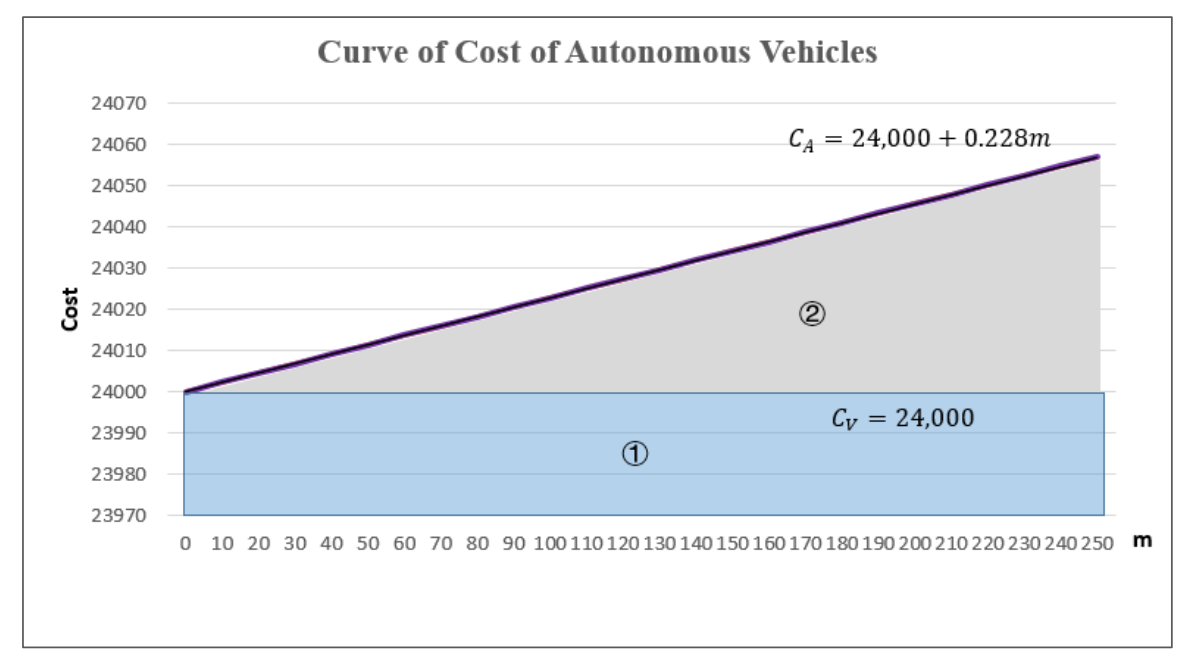

Figure 5. The curve of the cost of autonomous vehicles 
As can be seen from Figure 5, the total cost of using autonomous vehicles $\left(C_{A}\right)$ comprises two parts. Area (1) represents the fixed cost - the investment on purchasing autonomous vehicles $\left(C_{V}\right)$. Area (2) represents the accumulated operating cost, i.e., the accumulated cost of electricity consumption $\left(C_{E}\right)$, which is a variable cost that becomes large as the accumulated number of delivery $(m)$ increases. Therefore, the fixed cost $C_{V}$ contributes an initial value, and subsequent long-run incremental part of the total cost $\left(C_{A}\right)$ depends on the accumulated electricity consumption.

Similarly, the total cost of using manned trucks $\left(C_{M}\right)$ can be presented as Equation (11) through using the mean value of manned trucks' delivery time $\left(\overline{T_{M}^{*}}=19.5\right.$ mins $\left.\approx 0.33 h\right)$ as the input of the average time of per delivery $\left(\overline{T_{M}}\right)$ and putting other parameters' values in Table 1 into equation (6)-(8).

$$
C_{M} \approx m \times \overline{w_{M}} \times \overline{T_{M}^{*}}+\varphi \times m \times L \times \overline{P_{G}}=4.22 m+0.295 m=4.51 m
$$

The curve of Equation (11) is shown in Figure 6. The cost of a driver's wage $\left(C_{W}\right)$ and the accumulated cost of gasoline consumption $\left(C_{G}\right)$ are represented by area (3) and area (4) separately. Both $C_{W}$ and $C_{G}$ are variable costs that grow linearly as the accumulated number of delivery $(m)$ increases. Compared with $C_{G}$, the cost of a driver's wage $\left(C_{W}\right)$ is higher and contributes more to the growth of total cost $\left(C_{M}\right)$ in the long term.

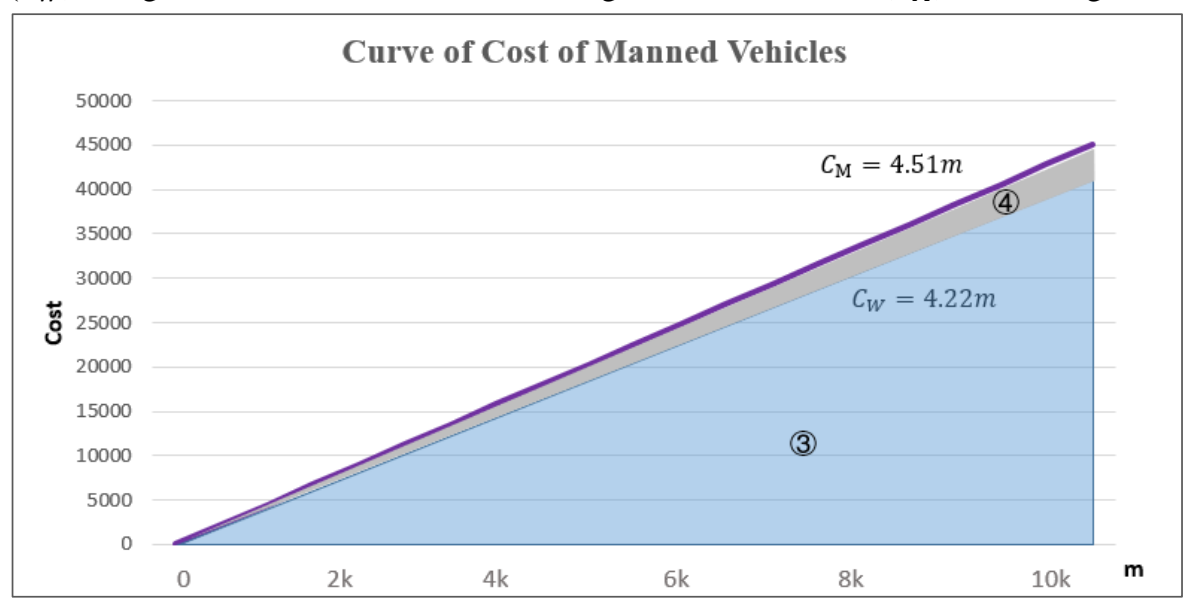

Figure 6. The curve of the cost of manned trucks

Putting the two curves into one coordinate system, Figure 7 shows that the two curves have an intersection with an abscissa $m^{*}=5604.86$.

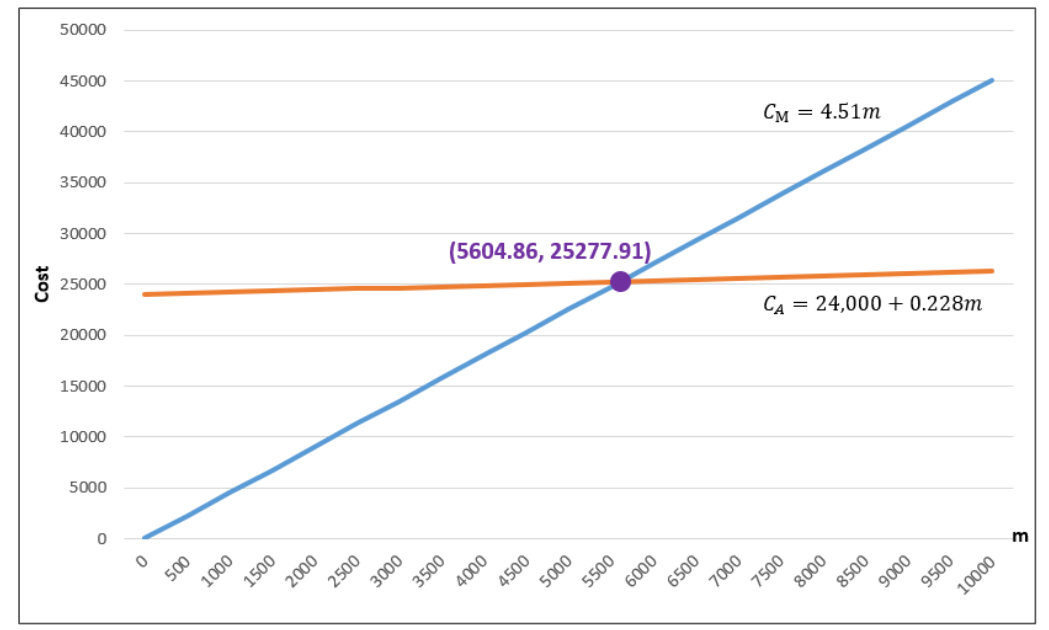

Figure 7. The combined curve for two costs 
Because $m$ represents the accumulated frequency of delivery, $m^{*}$ needs to be rounded to an integer, i.e., $m^{*}=5605$. As can be seen from Figure 7, the cost of using autonomous vehicles $\left(C_{A}\right)$ is significantly higher than that of using manned trucks $\left(C_{M}\right)$ for delivery on route S-A. But $C_{A}$ will be lower than $C_{M}$ after $5605\left(m^{*}\right)$ times of delivery, and the difference between $C_{A}$ and $C_{M}$ will continually enlarge. This means that using autonomous vehicles on route S-A will show cost-efficiency after 5605 times of delivery - approximately equivalent to 3 years if each vehicle deliveries 8 times a day, 250 working days a year.

Drawing the cost curves of electricity consumption $\left(C_{E}\right)$ and gasoline consumption $\left(C_{G}\right)$ in one coordinate system, Figure 8 shows that the cost of consuming electricity $\left(C_{E}\right)$ is only slightly lower than the cost of consuming gasoline $\left(C_{G}\right)$.

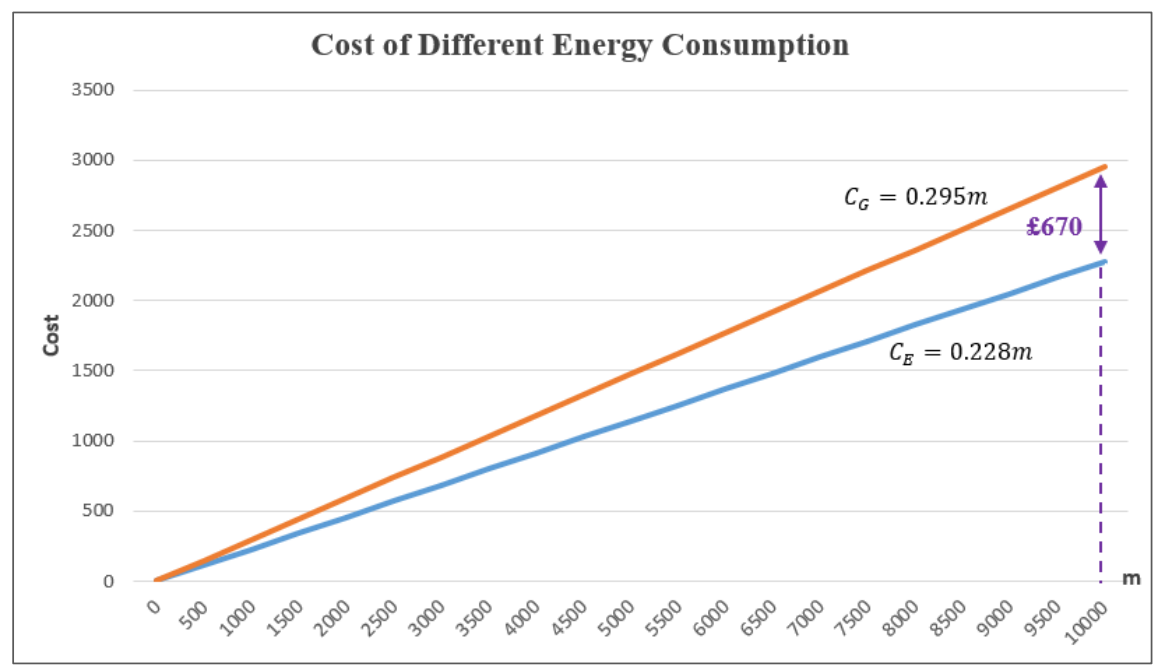

Figure 8 . The cost of different energy consumption

It can be seen that one autonomous vehicle that uses electricity will only save $£ 670$ than one manned truck that uses gasoline after 10,000 times of delivery - an extremely slight difference of nearly 7 pennies per delivery. Therefore, the cost advantage of autonomous vehicles, to a large extent, benefits from the vanishment of wages paid for drivers.

Based on the cost analysis, the research concludes that despite the significant initial investment in purchasing vehicles, using autonomous vehicles is more cost-efficient than using manned trucks for delivery on route S-A in the long term - nearly after three years since introducing autonomous vehicles. This cost advantage greatly depends on the removal of drivers and no need to pay for them commensurately. But the cost discrepancy due to fuel difference - autonomous vehicles using electricity while manned trucks using gasoline - is slight enough to be overlooked. The purchasing cost occupies a large part of the total cost of using autonomous vehicles for delivery. Therefore, the cost advantage of autonomous vehicles will appear more early in the future as the unit vehicle cost will become lower as a result of technological advance and commercialized production. However, purchasing vehicles will inevitably generate a considerable initial capital outflow, which may, in the short term, magnify the corporate leverage and cause a greater financial burden for institutions that are willing to replace current manned trucks with autonomous delivery vehicles.

\section{Conclusion}

This research fills the research gap - the lack of studies on the advantage of AVs for delivering goods from a dispatch centre to a pick-up station. This research analyses the economic feasibility of introducing autonomous vehicles for last-mile delivery by focusing on time and cost efficiencies. Although the last-mile delivery is an emerging industry with intrinsic complexity and uncertainty, this project thrusts the main research into the limelight of a single process - exclusively, the one-way route from one dispatch centre, i.e., the Sainsbury's supermarket, to one Amazon pick-up station at Coventry city - without considering the return route and any processes involving customers. In light of these, the cost and time of using autonomous vehicles have been reasonably estimated and compared with the status quo, i.e., using manned trucks. 
The research is designed in a way to accomplish all the project objectives identified at the beginning. Firstly, the delivery route is chosen and visualized through the Google map, which presents its distance and the number of crossroads. Then the time and cost models for using autonomous vehicles and using manned trucks are established separately, with both primary and secondary data as input for the values of parameters in these models. Based on these models, the time and cost spent by autonomous vehicles are calculated and compared with those of manned trucks to come to the findings on efficiency. For time efficiency, the key finding of this research is that using autonomous vehicles will spend more time than using manned trucks if the autonomous vehicles are limited to run on pedestrians or bicycle lanes at a commensurate low speed. However, it is infeasible for autonomous vehicles to run on motorways mixed with manned cars in the short term due to constraints such as technology immaturity, low social acceptability, and legal requirements. As for cost efficiency, the research concludes that there exists a breakpoint, after which using autonomous vehicles will become more cost-efficient than manned delivery trucks. But in the short term, the cost of autonomous vehicles is higher on account of the significant initial investment to purchase vehicles. Furthermore, the limitations of this research, the potential recommendations on how to improve the time and cost efficiency, and suggestions on further researchers are presented in the following paragraphs.

The analysis in this research is based on a set of idealized assumptions and commensurate models. While the research has some merits, it omits some important concerns that need to be addressed to substantiate the argument. Firstly, it is gratuitous to assume that one autonomous vehicle can carry the same weight as one manned truck. However, the real fact is that the carrying capacity of autonomous vehicles is usually significantly lower than that of manned trucks to satisfy the load limit for pedestrians or bicycle lanes. If so, the cost of using autonomous vehicles may become huger because a merchant needs to purchase more vehicles to achieve the same carrying capacity as a manned truck. Besides, the time and cost models in this research are oversimplified and overlook some important factors. The time models fail to consider the additional time, such as the delay caused by accidents, detours, or gridlock. The cost models do not include any implicit costs that emerged in line with the introduction of autonomous vehicles, such as the expense for training employees and for maintenance. These uncovered factors increase the uncertainty and ambiguity of the analysis results, and it may turn out that using autonomous vehicles is more time-efficient while less cost-efficient than using manned trucks. Furthermore, the models and findings in this research are not representative and cannot be applied to other business cases because the chosen route is highly unique and has a strong local identity. Whether using autonomous vehicles is more efficient depends on various factors such as the distance of a route, the number of traffic lights, the local speed limitation, the regional culture (Feng, 2020), etc., and these factors overlap and interact with each other in ways that cannot be detailed in this research. Such complexity makes it difficult for the conclusion of this project to be a universal principle that can be applied to the whole last-mile industry.

The project has thrown up many questions in need of further research. From this study, the cost and time efficiency of using autonomous vehicles for a particular single way is analysed. However, it is recommended that further research examine the cost and time efficiency for the whole last-mile delivery process that includes the loading, unloading, and return activities. Besides, the models built in this research is oversimplified, and, therefore, building unified time and cost models that can represent the most last-mile delivery processes could be usefully explored in further studies. Another possible area of future research would be to investigate the real distribution of parameters such as the waiting time at traffic lights and to build corresponding regression models for prediction. Although using random data that succumb to the normal distribution is one method of simulating the seemly out-of-order values of a parameter, more previous information would help us to establish a higher degree of accuracy on this matter. Furthermore, it would be interesting to assess the relationship and correlation between the time efficiency and the cost efficiency of introducing autonomous vehicles for last-mile delivery.

The findings of this study also have some important implications for future business practice. Unless the advanced technologies and local governments allow autonomous vehicles to run on regular motorways, the time efficiency of using autonomous vehicles for the last-mile delivery will not be attained. Due to the high purchasing cost at the beginning, supermarkets or warehouses can consider renting autonomous vehicles in the way of financial leasing. Besides, a merchant or a delivery company can introduce other alternative delivery methods to realize the time efficiency and the cost advantage. For example, unmanned delivery drones have lower unit costs, consume less energy, and fly faster. These advantages are conducive to achieving both cost and time efficiency (Lee et al., 2016).

\section{References}

Aqua-Calc. (2020). Cost to charge an electric car calculator. Retrieved from https://www.aqua-calc.com/calculate/electric-car-energy-cost 
Bloomberg. (2020). Driverless Delivery Vans Are Here as Production Begins in China. Retrieved from https://www.bloomberg.com/news/articles/2019-05-27/watch-out-mr-postman-robo-deliveries-are-now-real ity-in-china

Boysen, N., Schwerdfeger, S., \& Weidinger, F. (2018). Scheduling Last-mile Deliveries with Truck-based Autonomous Robots. European Journal of Operational Research, 271, 1085-1099.

https://doi.org/10.1016/j.ejor.2018.05.058

CNBC. (2015). Forget Delivery Drones, Meet Your New Delivery Robot. Retrieved from https://www.cnbc.com/2015/11/02/forget-delivery-drones-meet-your-new-delivery-robot.html

Dablanc, L., Giuliano, G., Holliday, K., \& O'Brien, T. (2013). Best Practices in Urban Freight Management: Lessons from an International Survey. Transportation Research Record, SAGE Journal, 22-44. https://doi.org/10.3141/2379-04

Energuide. (2020). What is the pump price? Retrieved from https://www.energuide.be/en/questions-answers/how-much-power-does-an-electric-car-use/212

Estonian World. (2016). Estonian delivery startup Starship and Mercedes-Benz team up to develop 'Robovans'. Retrieved from https://estonianworld.com/technology/starship-mercedes-develop-vans-filled-delivery-robots/

Feng, X. (2020). Managerial Challenges Faced by Goldman Sachs: From a Perspective of Human Resource Management. International Journal of Multidisciplinary Research and Publications (IJMRAP), 3(1), 10-17.

Gevaers, R., Van de Voorde, E., \& Vanelslander, T. (2009). Characteristics of innovations in last mile logistics using best practices, case studies and making the link with green and sustainable logistics. European Transport Conference, 2009 Proceedings, Leiden: Association for European Transport and contributors.

Global Petrol Prices. (2020). United Kingdom Gasoline prices, 30-Mar-2020. Retrieved from https://www.globalpetrolprices.com/United-Kingdom/gasoline_prices/

GOV.UK. (2020). Light signals controlling traffic. Retrieved from https://www.gov.uk/guidance/the-highway-code/light-signals-controlling-traffic

GOV.UK. (2020). Nation Speed Limit. Retrieved from https://www.gov.uk/speed-limits

Hoffmann, T., \& Prause, G. (2018). On the Regulatory Framework for Last-Mile Delivery Robots. Machines, 6(33), 1-16. https://doi.org/10.3390/machines6030033

Indeed. (2020). Truck Driver Salaries in the United Kingdom. Retrieved from https://www.indeed.co.uk/salaries/truck-driver-Salaries

James, Q., \& Katsuaki, T. (1984). Sample selection and cost underestimation bias in pioneer projects. (Unpublished Working Paper). California Institute of Technology, California, USA.

Joerss, M., Neuhaus, F., \& Schröder, J. (2016). How customer demands are reshaping last-mile delivery. Travel, Transport \& Logistics October 2016, Mckinsey \& Company.

Killeen, P. R. (2019). Predict, Control, and Replicate to Understand: How Statistics Can Foster the Fundamental Goals of Science. Perspectives on Behavior Science, 42, 109-132. https://doi.org/10.1007/s40614-018-0171-8

Kiss, A. (2019). Floods and Long-Term Water-Level Changes in Medieval Hungary. UK: Springer. https://doi.org/10.1007/978-3-319-38864-9

Koopman, P., \& Wagner, M. (2017). Autonomous Vehicle Safety: An Interdisciplinary Challenge'. IEEE Intelligent Transportation Systems Magazine, 9(1), 90-96. https://doi.org/10.1109/MITS.2016.2583491

Lee, J., Chang, H., \& Park, Y. (2018). Influencing Factors on Social Acceptance of Autonomous Vehicles and Policy Implications. 2018 Portland International Conference on Management of Engineering and Technology (PICMET), Porland. https://doi.org/10.23919/PICMET.2018.8481760

Lee, L. H., Chen, Y., Gillai, B., \& Rammohan, S. (2016). Technological Distribution and Innovation in Last-mile Delivery. (Unpublished working paper). Stanford Graduate School of Business, Stanford University, USA.

Li, B. (2013). A model of pedestrians' intended waiting times for street crossings at signalized intersections. Transportation Research Part B: Methodological, 51, 17-28. https://doi.org/10.1016/j.trb.2013.02.002

Mckinsey\&Company. (2019). A new look at autonomous-vehicle infrastructure. Retrieved from 
https://www.mckinsey.com/industries/capital-projects-and-infrastructure/our-insights/a-new-look-at-autono mous-vehicle-infrastructure

Moser, A., \& Korstjens, I. (2018). Series: Practical guidance to qualitative research. European Journal of General Practice, 24(1), 9-18. https://doi.org/10.1080/13814788.2017.1375091

Power Compare. (2020). Compare 2020 Electricity Prices: Average UK Rates \& Tariffs Per kWh. Retrieved from https://powercompare.co.uk/electricity-prices

Prause, G. (2019). Smart Contract for Smart Supply Chains. 9th IFAC Conference on Manufacturing Modelling, Management and Control MIM, 52(13), 2501-2506. https://doi.org/10.1016/j.ifacol.2019.11.582

Ranieri, L., Digiesi, S., Silvestri, B., \& Roccotelli, M. (2018). A Review of Last Mile Logistics Innovations in an Externalities Cost Reduction Vision. Sustainability, 10(782), 1-18. https://doi.org/10.3390/su10030782

Ryan, G. (2018). Introduction to positivism, interpretivism and critical theory. Nurse researcher, 25(4), 41-49. https://doi.org/10.7748/nr.2018.e1562

Slabinac, M. (2015). Innovation Solution for A 'Last-mile' Delivery - A European Experience. 15th international scientific conference Business Logistics in Modern Management, 111-129.

Stevens, L. (2015). Some Retailers Warn of Delivery Delays and Blame FedEx. The Wall Street Journal.

The New York Times. (2015). Skype Founders Build a Robot for Suburban Streets. Retrieved from https://www.nytimes.com/2015/11/03/science/skype-founders-build-a-robot-for-suburban-streets.html

Xia, H., \& Yang, H. (2018). Is Last-Mile Delivery a 'Killer App’ for Self-Driving Vehicles? Communications of the ACM, 61(11), 70-75. https://doi.org/10.1145/3239552

\section{Copyrights}

Copyright for this article is retained by the author(s), with first publication rights granted to the journal.

This is an open-access article distributed under the terms and conditions of the Creative Commons Attribution license (http://creativecommons.org/licenses/by/4.0/). 